\title{
Looking to the femoral rather than the carotid bifurcation to predict obstructive coronary artery disease?
}

\author{
Grigorios Korosoglou $^{1,2} \cdot$ Nasser Maylar ${ }^{3}$
}

Received: 16 July 2021 / Accepted: 17 July 2021 / Published online: 27 July 2021

(c) The Author(s), under exclusive licence to Springer Nature B.V. 2021

In this issue of the journal, Colledanchise et al. [1] compare the ability of ankle-brachial index (ABI) to that of plaque burden quantification in the femoral and carotid artery bifurcation for estimating the risk of obstructive coronary artery disease (CAD) by coronary angiography. The authors performed plaque burden quantification in femoral and carotid arteries and measures of the intima-media thickness (IMT) using vascular ultrasound as well as baseline ABI measures in 124 patients who were scheduled for coronary angiography due to suspected or known CAD. ABI, vascular ultrasound, and coronary angiography were all performed within the same day. Patients with acute coronary syndromes and previous femoral, carotid, or cardiac surgery were excluded from analysis. The presence of CAD was assessed categorically, i.e., the presence or absence of diameter stenosis $\geq 50 \%$ in at least one coronary segment, whereas CAD extent was assessed using a semi-quantitative score, based on the presence of single or multi-vessel CAD. For quantification analysis of plaque burden in femoral and carotid arteries, the maximal plaque height (MPH) and total plaque area (TPA) were assessed using axial and longitudinal planes of the right and left sided femoral and carotid

This commentary refers to the following paper https://urldefense. proofpoint.com/v2/url?u=https-3A_doi.org_10.1007_s105542D021-2D02334-2D9\&d=DwIGaQ\&c=vh6FgFnduejNhPPD0fl_ yRaSfZy8CWbWnIf4XJhSqx8\&r=dN6XQ0iN5osXbkB6Kb7T Bft0mqbXvGrDxJ5itSHl-pP9jMJpz2OMBjjLvMbu7tFw\&m= KCWRgt18rd_c7iyUUAkbwRLgSS2hvqkEtIWT1DsBaG0\&s= 7O21XvqU3RUzUGptGiy9zYCAK7h4hQreFRUGOu5dwqw\&e= .

Grigorios Korosoglou

gkorosoglou@hotmail.com

1 Department of Cardiology, Vascular Medicine \& Pneumology, GRN Hospital Weinheim, Weinheim, Germany

2 Cardiac Imaging Center Weinheim, Hector Foundation, Weinheim, Germany

3 Department of Cardiology I - Coronary and Peripheral Vascular Disease, Heart Failure, University Hospital Muenster, Cardiology, Munster, Germany arteries, respectively. The patient population exhibited high prevalence of hyperlipidemia (65\%), family history of CAD (71\%), and 79 (64\%) patients showed obstructive CAD. The authors reported that femoral TPA represents the most comprehensive estimate of atherosclerotic burden within two planes, exhibiting higher value for the detection of obstructive $\mathrm{CAD}$, compared to $\mathrm{ABI}$, IMD measures and to carotid TPA. Interestingly, femoral TPA exhibited the highest sensitivity for detecting significant CAD $(85 \%)$, whereas an abnormal ABI yielded low sensitivity of only $25 \%$ but the highest specificity of $91 \%$. After adjusting for body-mass index, glomerular filtration rate and atherogenic risk factors, femoral TPA was the most powerful parameter for the identification of significant CAD (Odds ratio $(\mathrm{OR})=11.9$, $\mathrm{p}<0.001$ ), followed by carotid MPH and TPA (OR of 4.46 and 3.48 , respectively $)$ and then by $\mathrm{ABI}(\mathrm{OR}=4.2)$, whereas both carotid and femoral IMD measures were not predictive.

The study of Colledanchise et al. [1] helps in better understanding the strengths and weaknesses of non-invasive measures, such as ABI and ultrasound-based carotid and femoral plaque burden for the estimation of the risk for underlying obstructive CAD. Both CAD and peripheral artery disease (PAD) are disorders exhibiting prevalence rates of 5-8\% and $10-20 \%$, respectively, depending on average age and geographic location of the studied populations [2]. In addition, based on data from the REACH registry [3] 18-35\% of patients with CAD and 46-68\% of patients with PAD, exhibit a so called polyvascular disease, with significant atherosclerosis in multiple vascular beds, including the heart, cerebrovascular vasculature, and the lower limb. Importantly, polyvascular disease is increasingly recognized as a particularly malignant constellation, requiring urgent clinical attention and aggressive medical treatment [4]. Overall, the socioeconomic burden of atherothrombotic disease is high and is expected to increase even more within the next years with an aging population and increasing prevalence of atherogenic risk factors, such as diabetes mellitus. 
From a technical point of view, ABI is a simple, noninvasive, time- and cost-effective parameter for the objective assessment of the functional state of the circulation in the lower limb, aiding the diagnosis of significant PAD. In addition, ABI is a well-established parameter for the estimation of future cardiovascular events, as demonstrated in large-scale, multi-center clinical trials [5, 6]. Although an $\mathrm{ABI}<0.9$ is a good indicator of progressed atherosclerosis, associated with poor cardiovascular outcomes, a normal ABI measure can be false negative in the presence of significant PAD in patients with media sclerosis due to type 2 diabetes mellitus. In addition, a normal ABI does not by any means exclude the presence of significant atherosclerotic disease or even polyvascular disease due to its low sensitivity. Thus, the prognostic ability of a normal ABI may therefore be limited in less advanced stages of atherosclerosis. Ultrasound of the carotid or femoral arterial beds, on the other hand, allows the direct visualization and if required the quantitative assessment of atherosclerotic plaque, which may be a better suited parameter for the identification of underlying atherosclerosis. Indeed, the quantification of total atherosclerotic plaque burden in the femoral bed using 2-dimentional (2D) ultrasound, was found to be the best predictor of obstructive CAD, beyond carotid atherosclerosis, IMD measures and $\mathrm{ABI}$ in the present study. Earlier studies have demonstrated a complementary prognostic value of femoral and carotid plaque burden for the risk stratification of lowrisk individuals [7]. In the same direction, the total number of diseased carotid and femoral artery segments were independent predictors of cardiac endpoints in men with different degrees of obesity and without known cardiovascular disease [8]. In the present study, the value of femoral plaque assessment was even higher than that of carotid atherosclerosis, so that cardiologists and vascular specialists might need to consider looking at these vessel segments too, when examining their patients for suspected CAD. In this regard, the current guideline issued in the year 2019 by the European Society of Cardiology for the management of hyperlipidemia newly recommended the assessment of carotid or/and femoral plaque burden by vascular ultrasound in patients at low or moderate risk, whereas the assessment of the IMD was not recommended any more [9].

However, several limitations need to be considered when interpreting the results of the present study. Thus, the study was conducted in high-risk, symptomatic patients already scheduled for invasive CAD assessment. Confirmation of these results in low-to-moderate-risk or even in asymptomatic patients would be of additive value in the diagnostic work-up and management of patients with suspected CAD. Furthermore, a cross-sectional design was used and unfortunately no follow-up data were reported, which is a limitation. Follow-up data would be necessary to establish the value of an ultrasound-based femoral plaque assessment approach, as a useful tool for the prediction of future cardiovascular events in such patients. In addition, the assessment of plaque was performed by $2 \mathrm{D}$ acquisitions and not by $3 \mathrm{D}$ ultrasound, which may further enhance the accuracy and reproducibility of plaque burden quantification in this context. Furthermore, the presence of a diameter stenosis $\geq 50 \%$ was selected as a criterion for the presence or absence of significant CAD by coronary angiography. As the authors acknowledge, X-Ray coronary angiography is not the ideal tool for assessing the extent of CAD. In this regard, the presence and extent of atherosclerotic plaque composition can nowadays be assessed non-invasively using calcium scoring measures and coronary computed tomography angiography (CCTA), the latter providing insights not only to the degree of lumen narrowing but also into the composition of atherosclerotic plaque and high-risk plaque features, as potential precursors of future acute cardiac events $[10,11]$. Moreover, established cardiac biomarkers, such as cardiac troponins were not available in this study. Troponins have been previously shown to be related to total atherosclerotic plaque burden and to high-risk plaque features in patients with chronic coronary syndromes [12] and their comparative value to vascular ultrasound for the diagnostic classification of such patients therefore remains to be evaluated in future studies.

Despite these limitations, the trial conducted by Colledanchise et al. [1] represents an important step towards better understanding the association between $\mathrm{ABI}$, femoral and carotid plaque burden by vascular ultrasound with the presence of obstructive CAD. Even if considered as hypothesisgenerating, the findings of the present study merit credit for demonstrating the ability of an easy-to-use ultrasoundbased approach for the estimation of CAD extent and severity. Future studies are now warranted to compare the role of vascular imaging for the diagnostic classification of patients with suspected CAD with that provided by cardiac biomarkers and CCTA and their impact on patients' management and clinical outcomes.

\section{Declarations}

Conflict of interest The authors declare that they have no competing interests.

\section{References}

1. Colledanchise KN, Mantella LE, Hétu MF et al (2021) Femoral plaque burden by ultrasound is a better indicator of significant coronary artery disease over ankle brachial index. Int J Cardiovasc Imaging. https://doi.org/10.1007/s10554-021-02334-9

2. Bauersachs R, Zeymer U, Brière J-B, Marre C, Bowrin K, Huelsebeck M (2019) Burden of coronary artery disease and 
peripheral artery disease: a literature review. Cardiovasc Ther 2019:e8295054

3. Smolderen KG, Wang K, de Pouvourville G, Brüggenjürgen B, Röther J, Zeymer U et al (2012) Two-year vascular hospitalisation rates and associated costs in patients at risk of atherothrombosis in France and Germany: highest burden for peripheral arterial disease. Eur J Vasc Endovasc Surg 43(2):198-207

4. Aday AW, Matsushita K (2021) Epidemiology of peripheral artery disease and polyvascular disease. Circ Res 128(12):1818-1832

5. Heald CL, Fowkes FGR, Murray GD, Price JF, Ankle Brachial Index Collaboration (2006) Risk of mortality and cardiovascular disease associated with the ankle-brachial index: systematic review. Atherosclerosis 189(1):61-9

6. Diehm C, Schuster A, Allenberg JR, Darius H, Haberl R, Lange $S$ et al (2004) High prevalence of peripheral arterial disease and co-morbidity in 6880 primary care patients: cross-sectional study. Atherosclerosis 172(1):95-105

7. Belcaro G, Nicolaides AN, Ramaswami G, Cesarone MR, De Sanctis M, Incandela L et al (2001) Carotid and femoral ultrasound morphology screening and cardiovascular events in low risk subjects: a 10-year follow-up study (the CAFES-CAVE study(1)). Atherosclerosis 156(2):379-387

8. Davidsson L, Fagerberg B, Bergström G, Schmidt C (2010) Ultrasound-assessed plaque occurrence in the carotid and femoral arteries are independent predictors of cardiovascular events in middle-aged men during 10 years of follow-up. Atherosclerosis 209(2):469-473

9. Mach F, Baigent C, Catapano AL, Koskinas KC, Casula M, Badimon L et al (2020) 2019 ESC/EAS Guidelines for the management of dyslipidaemias: lipid modification to reduce cardiovascular risk. Eur Heart J 41(1):111-188

10. Giusca S, Schütz M, Kronbach F, Wolf D, Nunninger P, Korosoglou G (2021) Coronary computer tomography angiography in 2021 - acquisition protocols, tips and tricks and heading beyond the possible. Diagnostics 11(6):1072

11. Korosoglou G, Chatzizisis YS, Raggi P (2021) Coronary computed tomography angiography in asymptomatic patients: still a taboo or precision medicine? Atherosclerosis 317:47-49

12. Korosoglou G, Lehrke S, Mueller D, Hosch W, Kauczor H-U, Humpert PM et al (2011) Determinants of troponin release in patients with stable coronary artery disease: insights from CT angiography characteristics of atherosclerotic plaque. Heart 97(10):823-831

Publisher's Note Springer Nature remains neutral with regard to jurisdictional claims in published maps and institutional affiliations. 